\title{
Bone Marrow Stem Cells Anti-liver Fibrosis Potency: Inhibition of Hepatic Stellate Cells Activity and Extracellular Matrix Deposition
}

\author{
Ervina Julien Sitanggang ${ }^{1, *}$, Radiana Dhewayani Antarianto ${ }^{2, *}$, Sri Widia A. Jusman ${ }^{3}$, \\ Jeanne Adiwinata Pawitan², Ahmad Aulia Jusuf ${ }^{2}$ \\ ${ }^{I}$ Department of Histology, Faculty of Medicine HKBP Nomensen, Medan, \\ Departments of ${ }^{2}$ Histology, ${ }^{3}$ Biochemistry and Molecular Biology, Faculty of Medicine Universitas Indonesia, Fakarta
}

Transplantation of bone marrow derived stem cells (BMSCs) has been reported inhibits liver fibrosis. Several in vitro studies by co-culturing BMSCs and hepatic stellate cells (HSCs) indirectly or directly in 2D models showed inhibition of HSC as the key player in liver fibrosis. In this study, we investigated direct effect of BMSCs on HSCs by co-culturing BMSCs and HSCs in 3D model as it represents the liver microenvironment with intricate cell-cell and cell-matrix interactions. Primary isolated rat HSCs and BMSCs were directly co-cultured at 1:1 ratio with hanging drop method. The monoculture of rat HSCs served as positive control. Mono-culture and co-culture samples were harvested on day 3, 5 and 7 for histological analysis. The samples were analyzed for extracellular matrix deposition by Masson's Trichrome staining, tenascin-C immunocytochemistry, resting HSC's state as shown by positive Oil Red O stained cells. Our results indicated $\mathrm{CD} 90^{+} \mathrm{CD} 34^{-}$BMSCs anti-liver fibrosis potency as evidenced by higher proportion of Oil Red O-positive cells in the co-culture group compared to the monoculture group and the significant decrease in extracellular matrix deposition as well as the decrease in tenascin- $C$ expression in the co-culture group $(p<0.05)$ compared to the monoculture group. These findings demonstrate that BMSCs have a potential therapeutic effect against liver fibrotic process through their capacity to inhibit HSCs activation and their effect in minimizing extracellular matrix deposition.

Keywords: Hepatic stellate cells, Bone marrow derived stem cells, Tenascin-C, Liver fibrosis, 3D co-culture

\section{Introduction}

Liver cirrhosis is a severe form of liver fibrosis.

Accepted for publication February 4, 2017, Published online May 30, 2017

Correspondence to Radiana Dhewayani Antarianto

Department of Histology, Faculty of Medicine Universitas Indonesia, Jl Salemba Raya no 6. Jakarta Pusat 10430, Indonesia

Tel: +62-21-3146129, Fax: +62-21-31900908

E-mail: radiana.dhewayani@ui.ac.id

*These authors contributed equally to this work.

(c) This is an open-access article distributed under the terms of the Creative Commons Attribution Non-Commercial License (http://creativecommons.org/ licenses/by-nc/4.0/), which permits unrestricted non-commercial use, distribution, and reproduction in any medium, provided the original work is properly cited.

Copyright (C) 2017 by the Korean Society for Stem Cells Research
Histological feature shows formation of regenerative nodules surrounded by fibrotic scar tissue. Chronic liver injury causes progression of liver fibrosis to liver cirrhosis. End stage liver disease is the bleak endpoint of uncompensated liver cirrhosis (1). Global Health Observatory of the World Health Organization in 2012 reported mortality rate caused by cirrhosis is 14,9 and 16 per 100.000 men in United States and UK, respectively. Mortality rate caused by cirrhosis in Indonesia is 52,7 per 100.000 men. This number is higher than US and UK (2).

Standard therapy for liver cirrhosis is liver transplantation. However, limited living liver donor and high cost remains a bottleneck problem for transplantation. A large number of patients died in the waiting list for liver donation (3). Experimental research for example injection of bone marrow derived stem cells (BMSCs) in liver cirrhosis patients 
indicates potential therapeutic option (4-6). Another experimental approach used hepatic stellate cells as target for anti-fibrotic therapy. Strategy for this approach utilizes chemicals to de-activate hepatic stellate cells or neutralizes fibrogenicity and proliferative capacity of hepatic stellate cells.

Hepatic stellate cell induces liver fibrosis. Activation of hepatic stellate cell into myofibroblast signifies the occurrence of fibrosis in liver tissue. Activated hepatic stellate cells displayed phenotypic and morphologic changes e.g. in proliferation capacity (7), chemotaxis, fibrogenicity, contractility and absence of retinoid vacuoles. These changes result in increased deposition of extracellular matrix proteins e.g. collagen, accelerated matrix turnover and scar tissue formation. Progressive liver fibrosis hampers normal hepatocyte functions thus resulting in a progressive liver failure $(8,9)$.

In vitro studies showed BMSCs effect on hepatic stellate cell's activity via paracrine factors (indirect co-culture using transwell) or direct co-culture 2D for juxtacrine effect $(10,11)$. Tenascin-C is one of the extracellular matrix protein that increases during liver fibrosis (12-14). Activated hepatic stellate cells are recognized as the cellular source of Tenascin-C $(15,16)$.

The objective of this study is to investigate the direct effect of BMSCs on HSCs by co-culturing BMSCs and HSCs in 3D co-culture model. 3D co-culture method could provide optimal visualization of intricate cell to cell, or cell to matrix interaction between BMSCs and HSCs.

\section{Materials and Methods}

Design of this study is in vitro experimental study using two cell populations : BMSCs from primary culture of Sprague Dawley (SD) rat bone marrow aspirate and HSCs from primary culture of SD liver parenchymal cells. The experiment was replicated 3 times. This study was performed from January to August 2015 at Institute of Human Virology and Cancer Biology (IHVCB) Faculty of Medicine Universitas Indonesia - Cipto Mangunkusumo Central Hospital (FKUI-RSCM), Histology Lab FKUI and Stem Cell Lab National Institute of Health Research and Development Ministry of Health Republic of Indonesia. Protocol of this study has been approved by Ethical Committee Faculty of Medicine Universitas Indonesia.

\section{Isolation and characterization of HSCs}

Isolation of HSCs was done according a to a modification of Riccalton-Banks method (17). In brief, rat liver was dissected into small pieces, incubated in collagenase
I for 30 minutes in $37^{\circ} \mathrm{C}$. The digested tissue was filtered using sterile steel filtration. The flow through cell suspension was centrifuged at $1200 \mathrm{rpm}$ for 10 minutes and supernatant was collected. Supernatant was centrifuged at $1200 \mathrm{rpm}$ for 10 minutes and pellet was collected. The pellet was resuspended with $6 \mathrm{~mL}$ medium (DMEM+FBS $10 \%+$ Penicillin/Streptomycin $1 \%+$ Fungizone $1 \%$ ). HSCs were characterized by morphology and culture well adhesiveness in addition by Oil Red O staining.

\section{Isolation and characterization of BMSCs}

Isolation of BMSCs was done according to a modification of Zhao method (18). In brief; femur and tibial bone were obtained. Top and bottom part of each bone was excised and discarded. $27 \mathrm{G}$ syringe was used to flush the bone marrow aspirate with PBS solution onto $10 \mathrm{~cm}$ petri dish. Bone marrow aspirate was collected and transferred to $15 \mathrm{ml}$ falcon tube. Further it was subjected to centrifugation at $1200 \mathrm{rpm}$ for 10 minutes. Pellet was collected and resuspended in $6 \mathrm{~mL}$ medium (DMEM+FBS $10 \%+$ Penicillin/Streptomycin 1\%+Fungizone 1\%). BMSCs phenotype was checked by flowcytometry analysis of CD90 and CD34.

\section{D co-culture of HSCs and BMSCs with Hanging Drop method}

This culture method used a $10 \mathrm{~cm}$ petri dish. $30 \mu \mathrm{L}$ mixture comprising of both cells suspension in ratio 1:1 $(\mathrm{v} / \mathrm{v})$ was carefully pipetted on to inner side of upper petri dish lid (open position). Each lid in the end contained of average 20 drops. The lower petri dish was filled with 5 $\mathrm{mL}$ of DMEM+FBS 10\%+Penicillin/Streptomycin 1\%+ Fungizone. With a single gesture, the upper lid was flipped to obtain closed petri dish position. The drops were hanging on the inner side of the upper lid. The dish was incubated in $\mathrm{CO}_{2}$ incubator at $37^{\circ} \mathrm{C}, \mathrm{CO}_{2} 5 \%$, humidity $95 \%$. There were four dishes. Three dishes according to day of harvest: day 3, day 5 and day 7. One dish was as negative control that contained harvested monoculture 3D hanging drop of HSCs at day 7.

\section{Oil Red 0 staining}

Inactive HSCs was determined by positive oil red O staining. Harvested hanging drops were collected day 3, day 5 or day 7 as well as negative control on day 7. Sample obtained was incubated with Oil Red O working solution (Histology FKUI, Indonesia) in eppendorf tube for 30 minutes. Stained sample was pipetted onto histology slide and observed under light microscope. Five high power fields were randomly selected from each sample. Oil red 
O positive cells were counted in each high power field. Mean of harvested sample on day 3, 5 or 7 were analyzed.

\section{Masson's trichrome staining}

Extracellular matrix deposition in the hanging drops was evaluated by Masson's Trichrome staining. Harvested hanging drops sample was collected day 3, day 5 or day 7 as well as negative control on day 7. Sample was pipetted onto histology slide. Masson's Trichrome staining (Histology FKUI, Indonesia) was performed and slide was observed under the light microscope. Five high power field was randomly selected from each sample.

3D co-culture hanging drop is an artificial structure. Prior report on Masson Trichrome staining of this structure is absent. Positive control of Masson Trichrome staining for collagen fibre in subcutaneous tissue showed histological feature of blue fibrous (wavy) streak appearance. This is not observed in the Masson Trichrome stained hanging drops. Thus we did visualization analysis of non-collagen type I ECM: lighter stained area indicated least abundant extracellular matrix deposition in comparison with darker stained area with Masson Trichrome's staining. We evaluated proportion of lighter stained area to whole drops area stained with Masson Trichrome (lighter and darker stained area). Lighter stained area in each high power field was measured using Optilab imaging software. Mean of harvested sample on day 3, 5 or 7 were analyzed.

\section{Immunochemistry tenascin-C}

Harvested co-culture hanging drops were collected on day 7 and negative control on day 7. Sample obtained was pipetted onto histology slide. Blocking endogenous peroxidase was done with $\mathrm{H}_{2} \mathrm{O}_{2} 3 \%$. Nonspecific binding reduction with donkey serum $1.5 \%$ blocking was done for 1 hour. Incubation with primary antibody anti-tenascin-C sc9871 (Santa Cruz Biotechnology, US) was done for 1 hour in moist chamber at room temperature. Incubation with biotinylated secondary antibody was done, followed by streptavidin-conjugated-peroxidase and DAB substrate addition. Nuclear counterstained with Hematoxylin Harris. Slide was observed under light microscope. Five high power fields were randomly selected from each sample. Areas of dark brown signals (positive tenascin-C) were measured in each high power field. Mean of dark brown signals in harvested sample on day 7 were analyzed.

\section{Data analysis}

Data analysis was performed using SPSS program. Number of inactivated HSCs, area of extracellular matrix deposition and tenascin $\mathrm{C}$ positive were analysed for normal distribution with Saphiro-Wilk test, followed by homogeneity Levene test. Comparison between groups was analyzed using independent t-test for parametric test or Mann-Whitney for non-parametric test. Statistical significance was considered at $\alpha=0.05$.
A

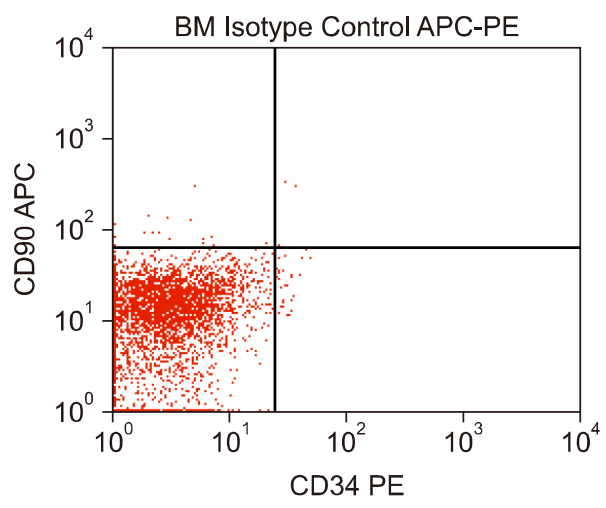

B

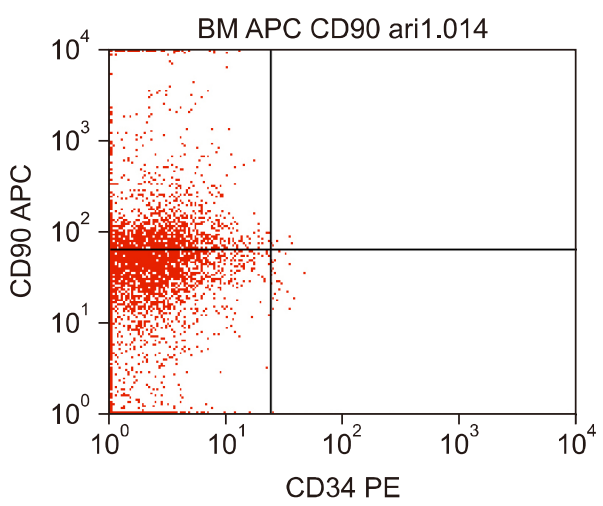

C

\begin{tabular}{crrrrrrr} 
Quad & Events & \% Gated & \% Total & X Mean & X Geo Mean & Y Mean & Y Geo Mean \\
\hline UL & 1,639 & 35.20 & 16.39 & 3.10 & 2.33 & 579.70 & 141.95 \\
UR & 4 & 0.09 & 0.04 & 30.22 & 29.89 & 77.50 & 77.21 \\
LL & 2,990 & 64.22 & 29.90 & 2.65 & 1.99 & 32.00 & 22.65 \\
LR & 23 & 0.49 & 0.23 & 29.15 & 28.76 & 30.74 & 25.08
\end{tabular}

Fig. 1. Phenotype of BMSCs. (A) Isotype Control APC-PE. (B) BMSCs stained with APC-labeled anti-CD90 and PE-labeled anti-CD34 antibody. (C) A table describing percentage of cell population in each quadrant in FACS blot. 


\section{Results}

\section{Phenotype analysis of BMSCs}

Flow cytometry analysis of BMSCs showed percentage of $\mathrm{CD} 90^{+} \mathrm{CD} 34^{-}$was $35.2 \%$. Isotype control was shown in Fig. 1A. This percentage was acquired from gated population in left upper quadrant (Fig. 1B). Description of other cell population was displayed in Fig. 1C.

\section{D co-culture BMSCs and HSCs effect on the proportion of inactive HSCs}

Staining results showed large extracellular oil red $\mathrm{O}$ droplets, which made it difficult to count the number of active and inactive HSCs. Therefore analysis was only done qualitatively. Qualitative observation indicated higher proportion of inactive HSCs in 3D co-culture compared to monoculture groups on day-3, day-5 and day-7 (Supple- mentary Fig. 1).

\section{D BMSCs and HSCs co-culture effect on extracellular matrix deposition}

Mean proportion of lighter stained area in 3D co-culture day 3 , day 5 or day 7 was lower than negative control (3D HSCs monoculture) (Fig. 2A, B). There was no statistical significant difference between $3 \mathrm{D}$ co-culture day 3 $(\mathrm{p}=0.116)$ or day $5(\mathrm{p}=0.781)$ with negative control. Comparison between 3D co-culture day 7 showed statistically significant difference with negative control (3D monoculture HSCs) $(p=0.01)$ (Fig. 2C).

\section{D BMSCs and HSCs co-culture effect on Tenascin-C expression}

Positive Tenascin-C expression was shown as dark brown signals in the extracellular area. Proportion of positive te-
A
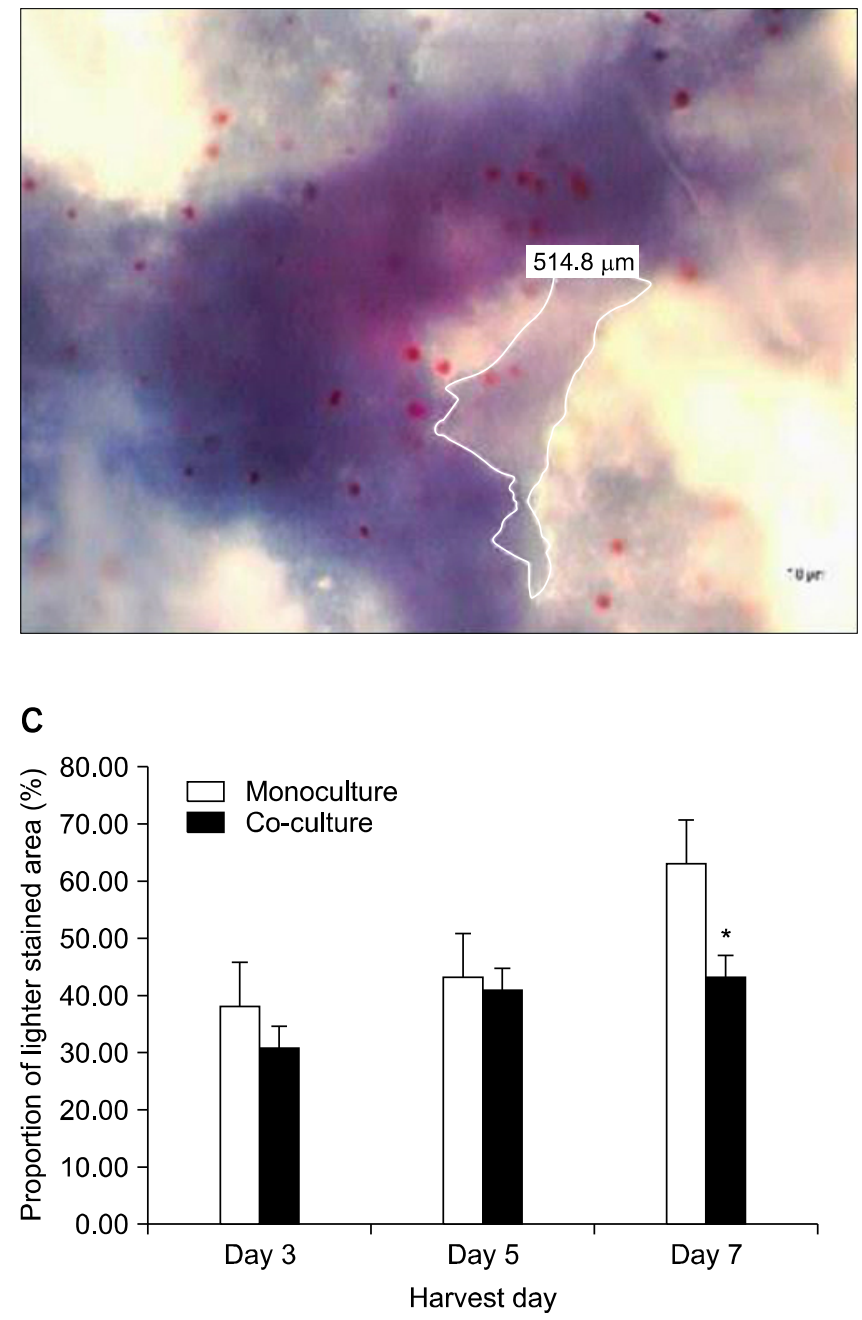

B

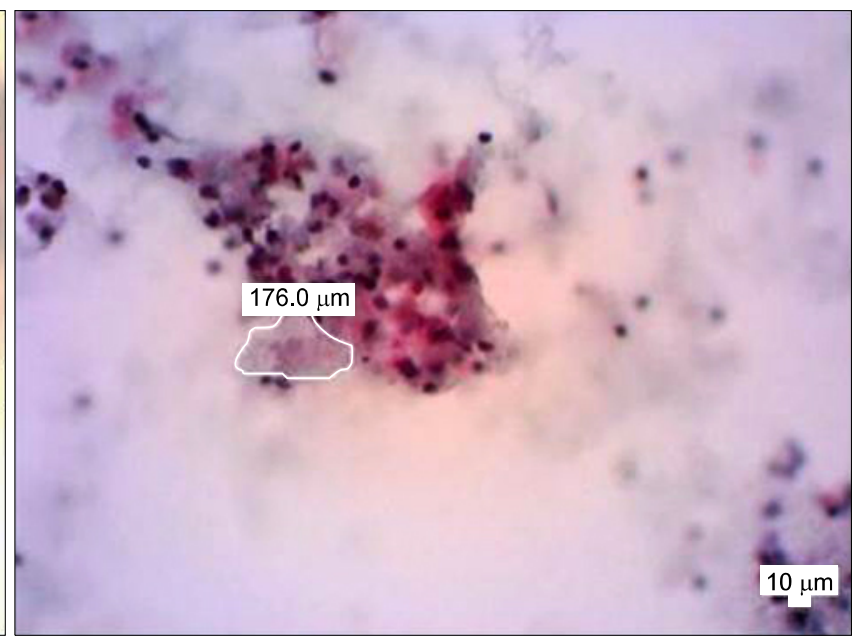

Fig. 2. Comparison of extracellular matrix deposition with Masson Trichrome staining (400× magnification). (A) Microphotograph Masson's Trichrome stained hanging drop mono-culture day 7 (400× magnification). (B) Microphotograph Masson's Trichrome stained hanging drop co-culture day 7 (400× magnification). (C) Mean proportion of lighter stained area which indicate least abundant extracellular matrix deposition. *Indicate significant difference between mean proportion of mono-culture 3D with co-culture $3 \mathrm{D}$. 
nascin- $\mathrm{C}$ area was evaluated by dividing area measurement of positive tenascin- $\mathrm{C}$ expression with whole hanging drop area in histology slide (Fig. 3A, B, C). Mean proportion of positive Tenascin- $\mathrm{C}$ area between $3 \mathrm{D}$ co-culture day 7 was statistically significant lower than 3D mono-culture $(p=0.004)$ (Fig. 3D).

\section{Discussion}

In vitro study to evaluate HSC activity can be intriguing. Culture well attachment has been reported to influence HSCs activity. Inactive phenotype was maintained either in suspension culture or non-adherent culture (21). Our hanging drop culture system provide qualitative technique to distinguish active and inactive HSCs with Oil red O staining. Our result suggested higher proportion of inactive HSCs in 3D co-culture BMSCs and HSCs compare to monoculture HSCs. Previous study using direct 2D co-culture HSCs and BM MSCs showed lower expression of activated HSCs (11). Increased HSCs apoptosis and decreased proliferation may cause lower proportion of activated $\operatorname{HSCs}(10,11,22)$.

Our result showed least abundant extracellular matrix deposition in 3D BMSCs and HSCs co-culture in comparison with 3D HSCs mono-culture. Decrease extracellular matrix deposition by 3D hanging drop co-culture BMDCs with HSCs provide evidence of liver anti-fibrotic effect. Parekkadan et al. showed pro-collagen type I peptide con-
A

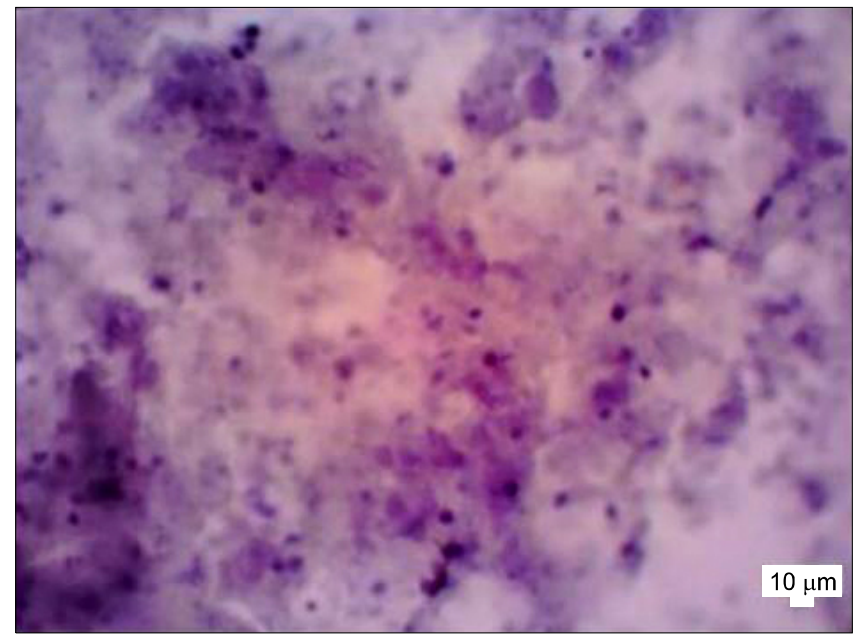

C

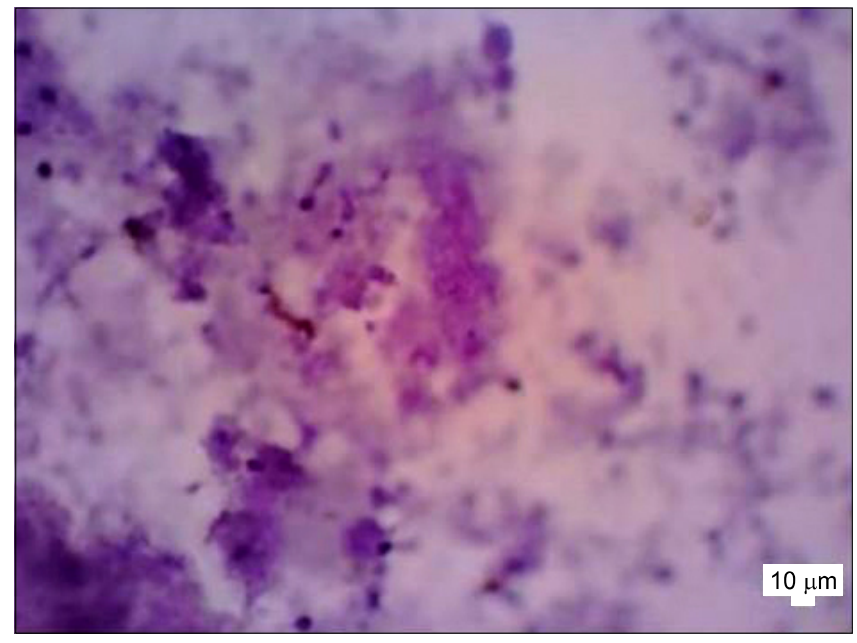

B

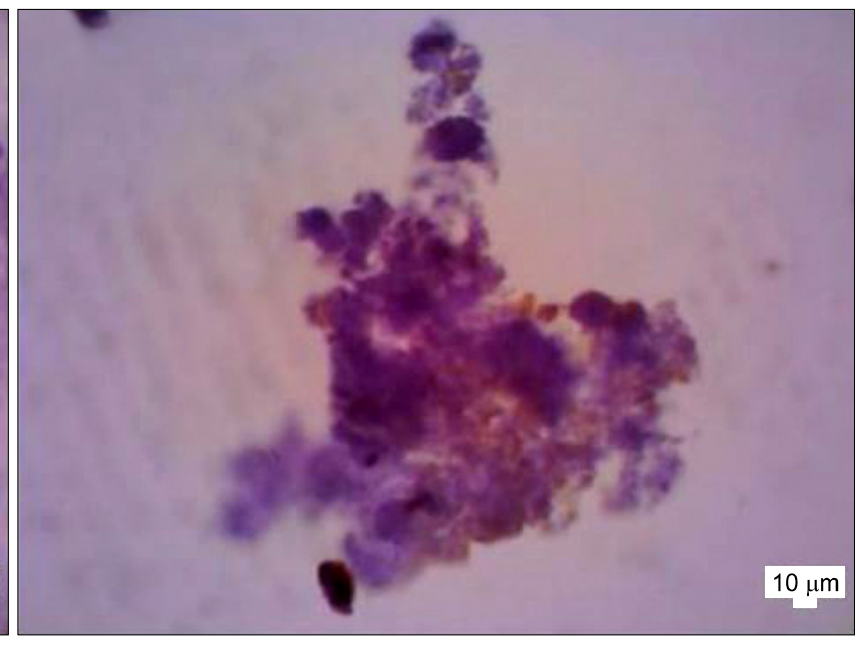

D

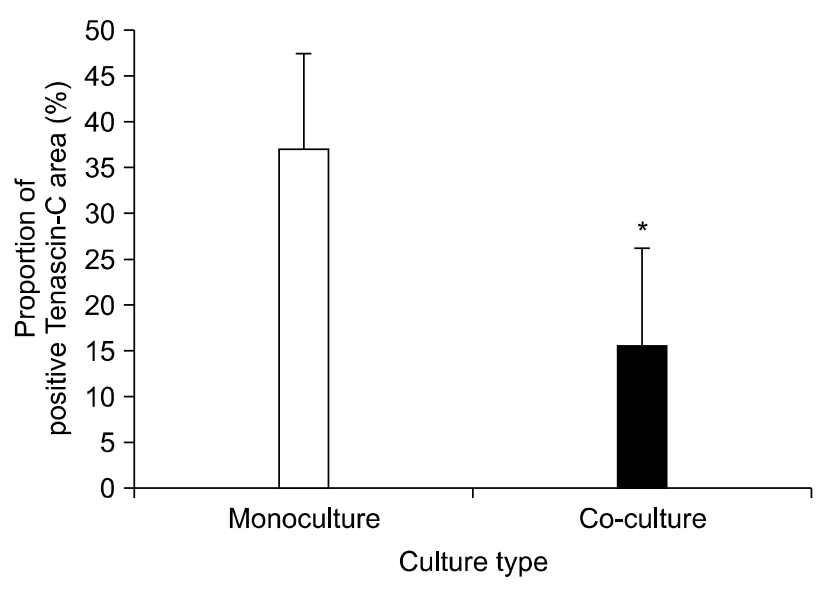

Fig. 3. Comparison between Positive Tenasicn $C$ area. (A) Microphotograph immunochemitry Tenascin-C negative control 3D mono-culture day 7, 400× magnification. (B) Microphotograph immunochemitry Tenascin-C 3D mono-culture day 7, 400× magnification. (C) Microphotograph immunochemistry tenascin-C 3D co-culture day 7, 400× magnification. (D) Mean of proportion of positive Tenascin-C area. ${ }^{*}$ Indicate statistical significant difference. 
centration was lower in HSCs co-culture with BM MSCs. BM MSCs inhibit collagen synthesis in activated HSCs by paracrine effect. BM MSCs secretes IL-10 and TNF- $\alpha$ (15).

Increased tenascin- $C$ expression -as one of the extracellular matrix protein secreted by activated HSCs- is associated with liver injury and fibrosis or cirrhosis (12-16, 23-25). Our result showed proportion of positive Tenascin-C area in 3D BMSCs and HSCs co-culture was lower than 3D HSC monoculture. Lower tenascin-C expression corresponds with higher resting HSCs or inactive HSCs. Prior studies by El Karef (12) and Jiang (16) showed activated HSC secretes higher amount of tenascin-C in comparison to resting HSCs. Tenascin-C plays a major role in increasing pro-collagen type I and III gene expression through several mechanism: robust inflammatory response by increased cytokine secretion, increased cell recruitment and increased TGF- $\beta$ (26).

Liver fibrosis study requires improved in vitro method from $2 \mathrm{D}$ co-culture to $3 \mathrm{D}$ co-culture for optimal microenvironment analysis. Our study uses 3D co-culture hanging drop method which formed cellular aggregates between two populations of cells. 3D culture system enable optimal cell to cell or cell to matrix interaction thus mimic the in vivo microenvironment $(19,20)$.

Limitation of this study was the low percentage of our isolated BMDCs phenotype compared to standard BM MSCs: $35.2 \% \mathrm{CD} 90^{+} \mathrm{CD} 34^{-}$vs over $80 \%$. Our study may initiate concomitant study to further refine isolation method with cell sorting technique. Another limitation of this study is difficulty in counting oil red $\mathrm{O}$ positive and negative cells. This fact arose due to the staining technique, which omit washing of stained cells hence the presence of extracellular oil red $\mathrm{O}$ droplets appeared.

Inhibition of HSC activity by direct interaction with BMDCs reduces fibrogenesis thus offer potential liver anti-fibrotic therapy. Further investigation is needed to elucidate the fibrogenic signaling pathways of liver microenvironment.

\section{Conclusion}

BMDCs with HSCs 3D co-culture provides in vitro evidence for least abundant extracellular matrix deposition, lower tenascin-C expression and suggested higher proportion of inactivated HSCs. BMDCs directly effect HSCs activation by reducing ECM deposition and tenascin-C secretion hence have liver anti-fibrotic potency.

\section{Acknowledgments}

This study is funded by PUPT Dikti 2015 research grant
(Hibah Penelitian Unggulan Perguruan Tinggi Universitas Indonesia). The authors acknowledge the work of Wahyunia LS in performing the culture and histology analysis and Ariyani $\mathrm{N}$ for performing the flow cytometry analysis.

\section{Potential conflict of interest}

The authors have no conflicting financial interest.

\section{Supplementary Materials}

Supplementary data including one figure can be found with this article online at http://pdf.medrang.co.kr/paper/ pdf/IJSC/IJSC-10-s16048.pdf.

\section{References}

1. Schuppan D, Afdhal NH. Liver cirrhosis. Lancet 2008;371: 838-851

2. Age-standardized death rates liver cirrhosis $(15+$ years $)$ per 100,000 population 2012 [Internet]. [cited 2014 Oct 14]. Available from: http:/gamapserver.who.int/gho/interactive_ charts/gisah/death_rates_cirrhosis/atlas.html.

3. Kim WR, Kremers WK. Benefits of "the benefit model" in liver transplantation. Hepatology 2008;48:697-698

4. Mohamadnejad M, Alimoghaddam K, Mohyeddin-Bonab $M$, Bagheri $M$, Bashtar $M$, Ghanaati $\mathrm{H}$, Baharvand $\mathrm{H}$, Ghavamzadeh A, Malekzadeh R. Phase 1 trial of autologous bone marrow mesenchymal stem cell transplantation in patients with decompensated liver cirrhosis. Arch Iran Med 2007;10:459-466

5. Ismail A, Fouad O, Abdelnasser A, Chowdhury A, Selim A. Stem cell therapy improves the outcome of liver resection in cirrhotics. J Gastrointest Cancer 2010;41:17-23

6. Levicar N, Pai M, Habib NA, Tait P, Jiao LR, Marley SB, Davis J, Dazzi F, Smadja C, Jensen SL, Nicholls JP, Apperley JF, Gordon MY. Long-term clinical results of autologous infusion of mobilized adult bone marrow derived CD34+ cells in patients with chronic liver disease. Cell Prolif 2008;41 Suppl 1:115-125

7. Kinnman N, Goria O, Wendum D, Gendron MC, Rey C, Poupon R, Housset C. Hepatic stellate cell proliferation is an early platelet-derived growth factor-mediated cellular event in rat cholestatic liver injury. Lab Invest 2001;81: 1709-1716

8. Elpek GÖ. Cellular and molecular mechanisms in the pathogenesis of liver fibrosis: An update. World J Gastroenterol 2014;20:7260-7276

9. Friedman SL. Hepatic fibrosis -- overview. Toxicology 2008;254:120-129

10. Parekkadan B, van Poll D, Megeed Z, Kobayashi N, Tilles AW, Berthiaume F, Yarmush ML. Immunomodulation of activated hepatic stellate cells by mesenchymal stem cells. Biochem Biophys Res Commun 2007;363:247-252

11. Chen S, Xu L, Lin N, Pan W, Hu K, Xu R. Activation 
of Notch1 signaling by marrow-derived mesenchymal stem cells through cell-cell contact inhibits proliferation of hepatic stellate cells. Life Sci 2011;89:975-981

12. Van Eyken P, Sciot R, Desmet VJ. Expression of the novel extracellular matrix component tenascin in normal and diseased human liver. An immunohistochemical study. J Hepatol 1990;11:43-52

13. Richter HB, Franke H, Dargel R. Expression of tenascin, fibronectin, and laminin in rat liver fibrogenesis--a comparative immunohistochemical study with two models of liver injury. Exp Toxicol Pathol 1998;50:315-322

14. Tanaka H, El-Karef A, Kaito M, Kinoshita N, Fujita N, Horiike S, Watanabe S, Yoshida T, Adachi Y. Circulating level of large splice variants of tenascin-C is a marker of piecemeal necrosis activity in patients with chronic hepatitis C. Liver Int 2006;26:311-318

15. Mekonnen GA, Ijzer J, Nederbragt H. Tenascin-C in chronic canine hepatitis: immunohistochemical localization and correlation with necro-inflammatory activity, fibrotic stage, and expression of alpha-smooth muscle actin, cytokeratin 7, and CD3 + cells. Vet Pathol 2007;44:803-813

16. El-Karef A, Kaito M, Tanaka H, Ikeda K, Nishioka T, Fujita N, Inada H, Adachi Y, Kawada N, Nakajima Y, Imanaka-Yoshida K, Yoshida T. Expression of large tenascin-C splice variants by hepatic stellate cells/myofibroblasts in chronic hepatitis C. J Hepatol 2007;46:664-673

17. Riccalton-Banks L, Bhandari R, Fry J, Shakesheff KM. A simple method for the simultaneous isolation of stellate cells and hepatocytes from rat liver tissue. Mol Cell Biochem 2003;248:97-102

18. Zhao DC, Lei JX, Chen R, Yu WH, Zhang XM, Li SN,
Xiang P. Bone marrow-derived mesenchymal stem cells protect against experimental liver fibrosis in rats. World J Gastroenterol 2005;11:3431-3440

19. Higgins CA, Richardson GD, Ferdinando D, Westgate GE, Jahoda CA. Modelling the hair follicle dermal papilla using spheroid cell cultures. Exp Dermatol 2010;19:546-548

20. Breslin S, O'Driscoll L. Three-dimensional cell culture: the missing link in drug discovery. Drug Discov Today 2013; 18:240-249

21. Friedman SL. Hepatic stellate cells: protean, multifunctional, and enigmatic cells of the liver. Physiol Rev 2008; 88:125-172

22. Qin S, Jiang H, Su S, Wang D, Liang Z, Zhang J, Yang W. Inhibition of hepatic stellate cell proliferation by bone marrow mesenchymal stem cells via regulation of the cell cycle in rat. Exp Ther Med 2012;4:375-380

23. Pas J, Wyszko E, Rolle K, Rychlewski L, Nowak S, Zukiel R, Barciszewski J. Analysis of structure and function of tenascin-C. Int J Biochem Cell Biol 2006;38:1594-1602

24. Jones FS, Jones PL. The tenascin family of ECM glycoproteins: structure, function, and regulation during embryonic development and tissue remodeling. Dev Dyn 2000; 218:235-259

25. Jones PL, Jones FS. Tenascin-C in development and disease: gene regulation and cell function. Matrix Biol 2000; 19:581-596

26. El-Karef A, Yoshida T, Gabazza EC, Nishioka T, Inada H, Sakakura T, Imanaka-Yoshida K. Deficiency of tenascin-C attenuates liver fibrosis in immune-mediated chronic hepatitis in mice. J Pathol 2007;211:86-94 